\title{
SOA-Based Telecom Product Management System Framework
}

\author{
Yanni Yuan, Bai Wang, Lei Zhang and Jianlin Wu \\ Beijing Key Laboratory of Intelligent Telecommunications Software and Multimedia, \\ School of Computer Science and Technology, Beijing University of Posts and \\ Telecommunications, Beijing 100876, P.R. China buptyyn@163.com
}

\begin{abstract}
By researching the telecom operations support system and the business support system, a telecom product management framework is presented via integrated with the customer management system (CRM), especially integrated with the analytic CRM and the business intelligence system (BI). It includes five layers, information resource object layer, metadata database layer, basic services layer, information services layer, and user interface layer. In addition, a metadata model is presented to solve the heterogeneous issues at the product data fields name, data type, meaning, database management system, etc. The diversified product information could be logical integrated via metadata service. And based on the unified product definition model, the sharing product analyzing information services could be provided in the whole enterprise angle of view. The framework function services are designed according to the Service Oriented Architecture (SOA), and implemented via Web services. The framework is a new try to improve the efficiency of enterprise macroscopic product analyzing, design, and information serving. It's has good flexibility, and expansibility in system function services.
\end{abstract}

Keywords: Product data management systems, Service-oriented architecture (SOA), Business information integration, Metadata, Business intelligence (BI), Customer relationship management (CRM)

\section{INTRODUCTION}

No product, no enterprise. The telecom company also is. Product management is very important work. Each of the China telecom company is a big group. A group is composed of headquarters and many provincial branches. And every branch usually has many subsystems of the telecom network operation support system (OSS) and business support system (BSS), e.g. CRM, Billing, business intelligence system (BIS), ERP, network management system, OA, etc. All these subsystems were built by many IT companies, so the IT architecture differences among the subsystem are unavoidable. Therefore the product information and customer information distributed in them are also hetero-structure in many faces. But from the company viewpoint, the product information model and the customer information model should be accordant. Usually, the product management includes five stages functions, product design, product pre-market analyzing, product post-market monitoring and evaluating,

Please we the following format when cining this chapter:

Yuan, Y., Wang, B., Zhang, L., Wu, J., 2007, in IFIP International Federation for Information Processing, Volume 255 , Research and Practical Issues of Enterprise Information Systems Il Volume 2, eds. L. Xu, Tjoa A., Chaudhry S. (Boston: Springer), pp. 1251-1260. 
product optimizing and the lasted product eliminate through selection or contest in telecom. Product design and analysis require the analysis results in CRM of the customers who ordered the product, and in the same way product is a key customer subdivision analysis dimension in CRM, thus the product management and the CRM are supplement each other and indivisible. But now the CRM or BIS are mainly built on the different middleware system, e.g. CORBA, EJB, etc. CORBA, EJB, or other middleware technique frameworks mostly are tightly coupling. The information interchange and service function sharing have many difficulties among them. [1]

In SOA, the services is a well-defined and invoke-able unit of business function, accesses business data or facilitates a change of business data from one valid and consistent state to another[2]. SOA enables flexible connectivity of applications or resources by representing every application or resource as a service with a standardized interface, then enabling them to exchange structured information or other messages, mediating the message exchange through an Enterprise Service Bus (ESB). ESB could be view as a logical architectural construct, provides interconnectivity services. The SOA infrastructure is also used to facilitate the management of business performance and quality of service, enable the companies to build stronger, more profitable customer relationships [3-5].

So based on the SOA, the telecom product management system framework (TPMS) is presented. Firstly, the product information is integrated via product structure metadata service, semantic metadata service, access metadata service. Then product analyzing information service is designed to satisfy the different product phase information requirement, e.g. product definition information service, product life cycle analyzing service, etc. TPMS follows the web service technology standard series, and the product analysis services semantic description information is enriched via using semantic web technology for reference [6-8].

The remainder of this paper is organized as follows. Section 2 reviews several related research works on product information management system and presents a unified product definition model via UML. Section 3 defines the TPMS framework, and presents the system function services design. Section 4 addresses some key issues, and uses a simple information access request as a case study of cooperation among the services in this new infrastructure. A brief conchusion and future works are addressed in Section 5.

\section{PRELIMINARIES}

\section{I Problem Description}




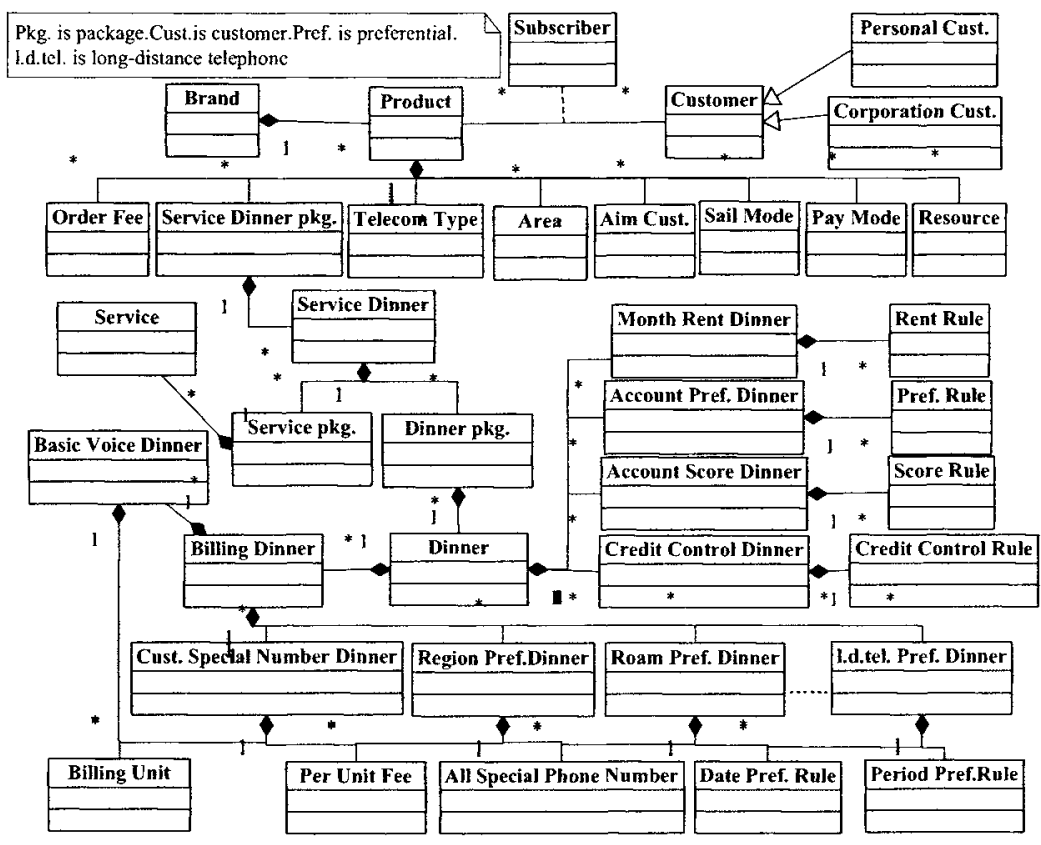

Figure 1. Telecom Product Definition Model

In China, different telecom enterprise has different product concept definition. In one enterprise group, the product information management in different branch's CRM or other system is mostly not accordant. So, to implement the holistic product design and management, the first step should give a consistent and clear definition of the product and other telecom domain shared concepts. The following four is the kernel telecom enterprise information object entity. As shown in Figure 1.

Telecom product, is a basic product unit or a set of basic product unit could be ordered by customer. A basic product unit is usually composed of market aim definition attributes, billing rules, accounting rules, customer using services, and diversified preferential rules, etc. A customer can order one or more products.

Customer, is a natural, physical entity. It could be a person or a company. Telecom enterprise usually designed different kind of products to satisfy the different customer group.

Subscriber, is a logical existent entity. When a customer ordered a product, a subscriber exists. A customer can has one or more subscribers. The state of subscriber is changed according the customer consuming behavior, e.g. when his or her arrearage goes beyond the credit limit, the state should be changed to stop by OSS.

Service, is a concept different from the Web service or the service in SOA. Here, it is not a technique word, but a telecom operation word, e.g. the local voice service, the 
long distance voice service, the short message service, etc. It is the key component of the product. In addition, the service also indicates the customer care contact service. Many services usually are integrated as a service package in a product. The cost of the service is defined in the diversiform dinners, e.g. month rent cost dinner, account preference dinner, and call billing dinner, etc.

\subsection{Telecom Product Definition Model}

Based on the composing analyzing of many kind of telecom product, the telecom product definition generally includes nine categories information [9]. As shown in Figure 1.

- Brand information describes the product is included in which brand kind.

- Order fee describes what fee should be pay when customers order it.

- Service dinner package describes the product how to billing and accounting.

- Telecom type describes the product is which telecom network, CDMA, GSM, or others.

- Area describes the product is designed for what market sale area.

- Aim customer definition describes the product is designed for what character customer.

- Sale mode describes customer could choose what kind of sale method, e.g. band a mobile phone and prepay some money or promises a number of on net months.

- Pay mode describes customer how to pay their bill, prepay or post-pay.

- Resource describes the product bind what resources, e.g. phone number, SIM/UIM card, or others.

\section{SOA-BASED TPMS FRAMEWORK}

\subsection{Two Typical Scenarios}

What does TPMS look like? Let us look at two scenarios. Here we take product basic definition information request for example. Normally, the enterprises market department takes charge of the macroscopic product brand programming. And different branch market departments bear responsibility for detail product design and market sale monitor according the headquarters criterion.

A Traditional product management Scenario. If the headquarters want to gather the main sale products in all branches for analyzing the brand strategy rationality and researching its marketing effect, it needs to inform each branch manually in traditional OSS/BSS system. And when accept the requirement message, each branch collects, summarizes, and reports the key business information to the headquarters manually. After HQ accepts all these information, it still needs to tidy them. 
Sometimes misapprehend is inevitably. So the process is low efficiency and not facility.

A TPMS Scenario. The same question in the TPMS, no need each branch to manually collect and send their products information to the HQ, only need the HQ user to $\log$ on the TPMS and download the concerned product information of all branches, and the information about them is good format, uniform and consistent. Obviously, the method is advantageous, convenient, and high efficiency.

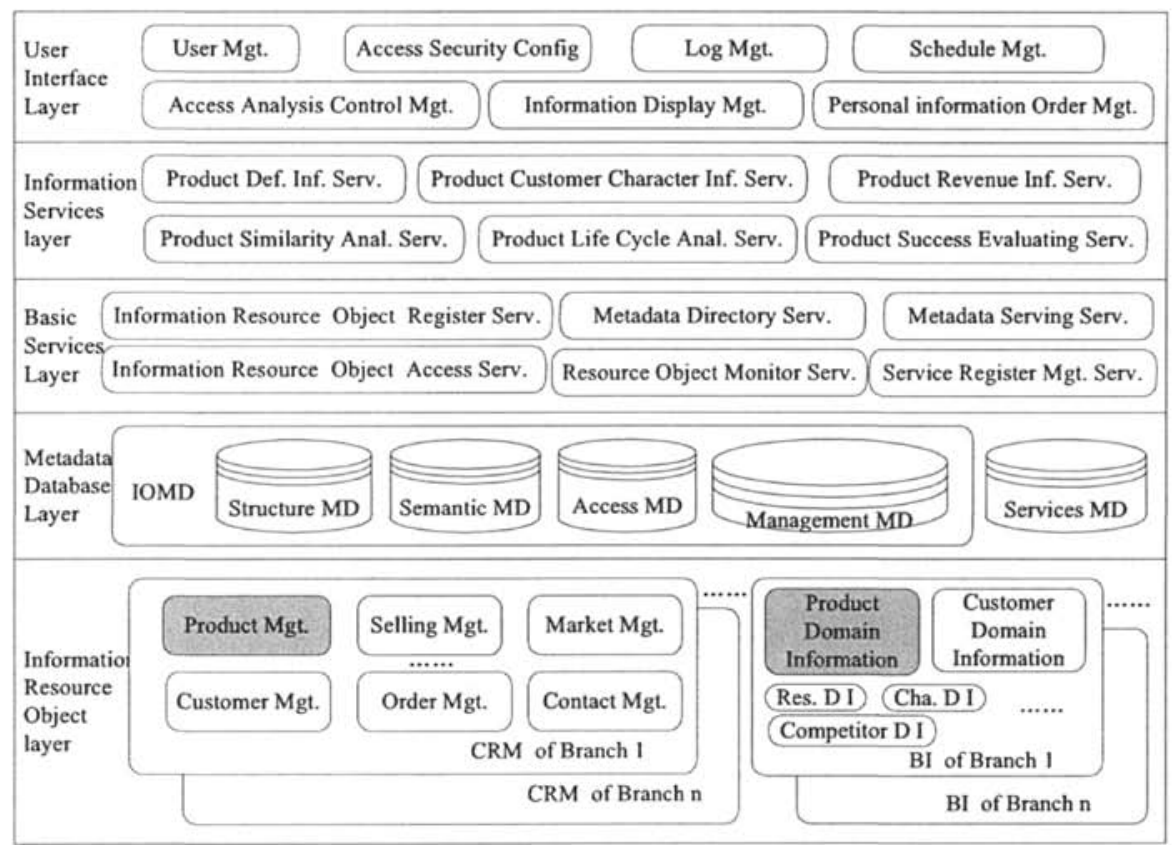

Figure 2. SOA-based TPMS Framework

\subsection{Framework Definition}

The TPMS framework could be described by a five element set: $\mathrm{F}=(\mathrm{IRO}, \mathrm{MDB}$, BS, PIS, UI ). And the five elements respectively denote the framework five layers from bottom to top $[10,11]$. As shown in Figure 2. IRO (Information Resource Object Layer). The product definition information mainly stores in the each branch CRM system. The consumer bill and pay analyzing information of the products is stored in the BI system. So the TPMS needs to integrate all these information distributed in each CRM and BI. CRM still includes market management, customer management, order management, contact management, etc. BI also includes customer analyzing domain information, resource analyzing domain information, channel analyzing 
domain information, competitor analyzing domain information, etc. If product management needs these information, they could be likewise convenient integrated.

- MDB (Metadata Database Layer). The metadata in TPMS includes information object metadata and framework function service metadata. In addition, the information object metadata can be classified four kinds. Structure metadata (STMD) defines the data store format, e.g. field name, data type, data precision, etc. Semantic metadata (SEMD) defines the data meaning. Access metadata (ACMD) defines the data location. Management metadata (MAMD) defines the data create and update information.

- BS (Basic Services Layer). This layer is mainly charge for registering the IRO metadata to the MDB, and searching the metadata about the information access demand via metadata service. The IRO could be shared by other system, none but registered to $\mathrm{MDB}$.

- PIS (Information Services layer). This layer is the TPMS main predominance than other system. Based on the different information requirements in different telecom product life cycle stage, the special product analyzing information services are designed. At the same time, information service could be flexible added according the demand.

- UI (User Interface Layer). This layer is the interface between the system and user. It includes system user management (UM), access security configure management (ASCM), log management (LM), access analysis control management (AACM), schedule management (SM), information display management (IDM), personal information order management (PIOM). AACM takes charge of response to the user information requirement and invoke different information services cooperating to gain the results.

\subsection{Framework Services Design}

The TPMS key services mainly in the basic services layer and information services layer. These services' function is independently described as following.

- Basic services layer

- Information resource objects register service (IRORS) is responsible for registering the IRO to the MDB.

- Information resource objects access service (IROAS) is responsible for building the connection between the information service and the IRO store location, then accessing the data.

- Resource objects monitor service (ROMS) is responsible for monitoring the shared IRO state and updating the metadata in MDB.

- Service register management service (SRMS) is responsible for all technique services unified management via UDDI.

- Metadata directory service (MDS). The metadata items congregate and form the metadata catalog. MDS is responsible for maintaining the metadata item, e.g. adding new items, updating or deleting old items.

- Metadata serving service (MSS) is responsible for search the metadata directory for the pre-described four kind metadata about the requested information. 
- Information services layer

- Product definition information service (PDIS) is responsible for search the nine faces definition information about the request concerned products.

- Product customer character information service (PCCIS) is responsible for search the character information about the customers who ordered the request concerned products.

- Product revenue information service (PRIS) is responsible for search the revenue information about the request concerned products.

- Product similarity analyzing service (PSAS) is responsible for analyzing the similarity about the request concerned products.

- Product life cycle analyzing service (PLCAS) is responsible for evaluating the life cycle stage about the request concerned products.

- Product success evaluating service (PSES) is responsible for evaluating the market sale success degree about the request concerned products.

The services are defined using explicit interfaces, independent of service implementations, and the contract between service requestors and service providers is unnecessary as in a group. They are invoke-able through common communication protocols, and location transparency. Services are described using a standardized interface via service metadata defining the service, its operations, preconditions, input, output, parameters and effects, how the service is reached and its location. Input and outputs may be simple parameters or XML documents.

SOA provides the flexibility required to operate in an On-Demand Environment. Web Services specifications is the implementation of SOA features. Many Web Services specifications are being standardized. IBM and other vendors have implemented these specifications, anticipating the standards. The Web Service function stack is rich and getting richer. The WSDL (Web Services Description Language), SOAP (Simple Object Access Protocol), and UDDI (Universal Description, Discovery, and Integration) are basic three among them. And the WSDL is an XML document that describes a Service using a number of key elements $[12,13]$. The framework service metadata design should reference WSDL and add some new needed describing element, e.g. product information service semantic category.

\section{KEY ISSUES OF THE FRAMEWORK IMPLEMENTATION}

TPMS needs to dynamic share the product information from the geographically distributed product information store subsystem, and provides a unite access interface for user, so as to these product information stored in one system. For the goal, some key issues must be solved.

\subsection{Telecom Product Meta-model Definition}

The telecom product meta-model is presented for uniform the product information management. As shown in Figure 3. The meta-model only contains the STMD about 
the database, table, and field. It does not include other database object type, e.g. key, store process, index, etc. And the basic SEMD, ACMD, MAMD, Web service metadata model element are presented. Based on the meta-model, three typical kinds of hetero- structure questions could be resolved $[14,15]$.

Name consistency. Same meaning fields though having different name could be endowed a same semantic name to unify them. Different meaning fields despite having same name could be endowed different semantic name to distinguish them.

- Meaning consistency. Same product information dimension code exists different meaning, e.g. about gender dimension code, ' 10 ' denotes male in a system, but ' 10 ' denotes female in another system. It could be resolved by map the different system code to the unified semantic code model definition.

- Access consistency. Access hetero-structure is transparent for user via system collecting information from different location.

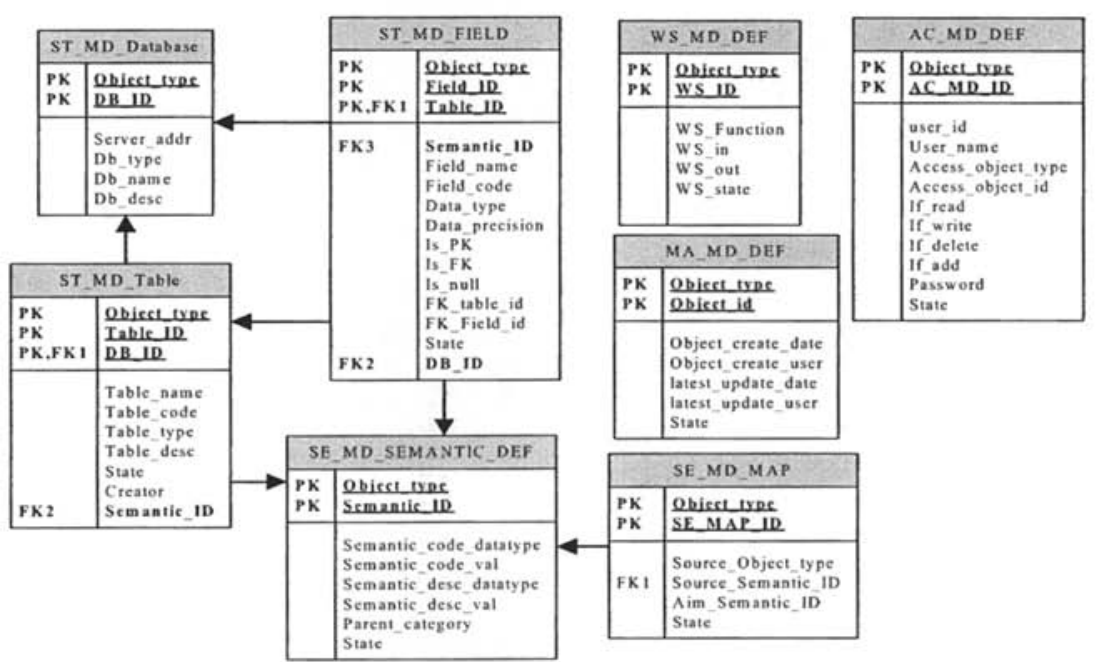

Figure 3. Telecom Product Meta-model Definition

\subsection{Product Information Resource Object Register and Share Mechanism}

In the framework the product information resource object register and share mechanism is as shown in Figure 4.

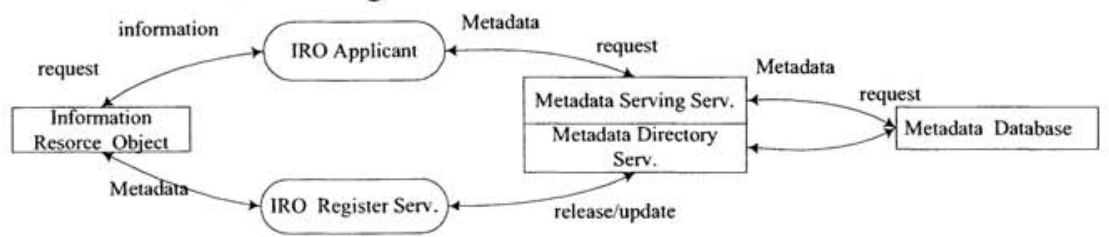

Figure 4. Product IRO Register and Share Mechanism 
IRO must be registered to the metadata database via IRO register service invoking the MDS. Then it could be shared by system users via MSS to search.

\subsection{Product Information Resource Object Register and Share Mechanism}

When an information requirement is brought forward, the services should be invoked and cooperate with each other. The key problem about how to satisfy the requirement is to ascertain the relevant services task sequence. Information service metadata needs to describe the cooperation sequence among the basic services. Here we take a simple product definition information request for example. As shown in Figure 5. The service function is explained in Section 3. Here, the metadata serving service is subdivided to structure metadata service, semantic metadata service, accessing metadata service.

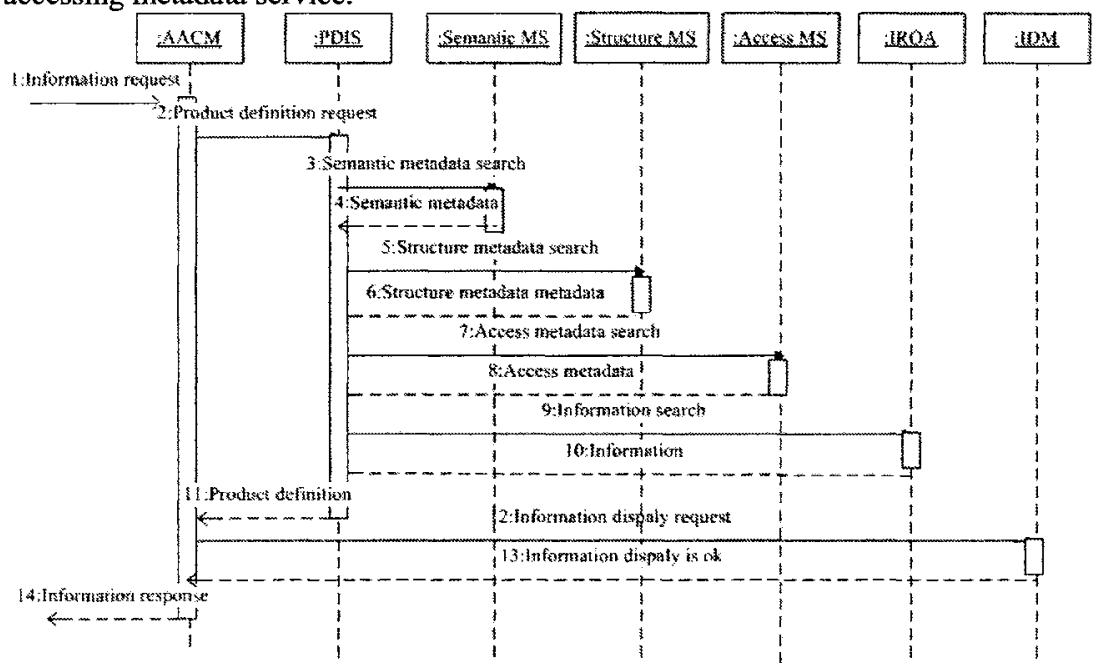

Figure 5. The Cooperation among the Framework Services

\section{CONCLUSIONS}

This paper presented a new paradigm for telecom product management system, which consists of a dynamic collection of geographically distributed product information store OSS/BSS subsystem. User can browse the product information in different branch system as if they are stored in one system.

TPMS makes it possible for the HQ or branch product managing user to get a convenient access to all product information resources in the whole enterprise through a local interface. They could conveniently get the product analyzing information. The registered metadata give a global view of the enterprise product information. The framework has good expansibility and gives a feasible method to resolve the telecom 
product information and service share problem, and reduce the system costs by share the product information services among the branches. TPMS can radically improve the efficiency on the product analyzing and design work, and reduce the information collecting manual work. In the next step, we need to study the combination and collaboration process of basic information services for supporting more flexible, complicated information service.

\section{REFERENCES}

1. J.K. Wang, B. Wang, and B. Ai, The Service-Scenario Software Architecture Pattern of OSSPBSS, Journal of Beijing University of Posts and Telecommunications. Volume 27, pp.30-38, (2004).

2. T. Zhang and S. Ying, Description of Service-oriented Architecture Based on UML, Computer Engineering. Volume 31, Number 16, pp.89-91, (2005).

3. M. Wang, J. LI, Z. Fan, and L. Xu, A Service Model for Virtual Resource Management and Its Implementation, Chinese Journal of Computer. Volume 28, Number 5, pp.856$863,(2005)$.

4. Z. Ma and H. Chen, A Service-Oriented Architecture Reference Model, Chinese Journal of Computer. Volume 29, Number 7, pp.856-863, (2006).

5. H. Shao and J. Kang, Research and Application of Enterprise Service Bus, Computer Engineering. Volume 33, Number 2, pp.220-222, (2007).

6. J. Li, S. Chen, and H. Ma, Research of Service-oriented Architecture Reference Model and Its Application, Computer Engineering. Volume 32, Number 20, pp.100-102, (2006).

7. X. Yang, L. Xu, Y. Dong, and Y. Wang, Web Service Description and Discovery Based on Semantic Mode, Wuhan University Journal of Natural Sciences. Volume 11, Number 5, pp.1306-1310, (2006).

8. H. Li, H. Song, and L. Jiao, Study of Service-oriented Architecture in Large-scale Database System, Computer Engineering. Volume 33, Number 2, pp.85-87, (2007).

9. X. Fu, S. Li, and M. Guo, Rules for Ontology Level Expression in Product Data, Journal of Computer Aided Design \& Computer Graphics. Volume 17, Number 6, pp.1159-1166, (2005).

10. T. Pollet, G. Maas, J. Marien, and A. Wambecq, Telecom Services Delivery in a SOA, in Proc. of 20th International Conference on Advanced Information Networking and Applications (AINA.2006), Volume 2 (IEEE., Vienna, Austria, 2006), pp.21-26.

11. A. Mocan, M. Moran, E. Cimpian, and M. Zaremba, Filling the Gap-Extending Service Oriented Architectures with Semantics, in Proc. of International Conference on eBusiness Engineering (ICEBE'06) (IEEE., Shanghai, China, 2006), pp.594-601.

12. T. Zhang, S. Ying, S. Cao, and X. Jia, A Modeling Framework for Service-Oriented Architecture, in Proc. of the Sixth International Conference on Quality Software (QSIC'06) (IEEE, Beijing, China, 2006), pp.219 - 226.

13. D.K.W. Chiu, P.C.K. Hung, and K.K.K. Lee, Web-Service Based Information Integration for e-Financial Planning System Matchmaking Decision Support, in Proc. of International Conference on Web Intelligence and International Agent Technology Workshops (WI-IAT 2006) (IEEE, Honk Kong, China, 2006), pp.259-262.

14. D. Linner, J. Radusch, S. Steglich, and S. Arbanbowski, Request-driven Service Provisioning, in Proc. of SICE-ICASE International Joint Conference (IEEE, Bexco, Korea, 2006), pp.2612-2617.

15. X. Li, Y. Yang, and W. Guo, Data Share-and-exchange Platform Based on ESB, Computer Engineering. Volume 32, Number 21, pp.217-223, (2006). 